\title{
Innovative planning synergies between manufacturing processes and microgrids
}

\author{
C. Gamarra ${ }^{1}$, M. Ortega ${ }^{1}$, E. Montero ${ }^{1}$ and J.M. Guerrero ${ }^{2}$ \\ ${ }^{1}$ Department of Electromechanical Engineering, University of Burgos, Spain \\ E.P.S., Avenida Cantabria s/n, 09006 Burgos (Spain)
}

Phone/Fax number:(+34) 9472589 00, e-mail: cgamarra@ubu.es, moizquierdo@ubu.es, emontero@ubu.es

${ }^{2}$ Department of Energy Technology, Aalborg University, Denmark

Pontoppidanstraede 101, room 79, 9220 Aalborg East, Denmark

Phone: (+45) 99409726 , email: joz@et.aau.dk

\begin{abstract}
Industrial equipment is evolving towards data gathering and communication, following the Internet of things approach. During last years, different management systems and related standards have been developed, in order to raise the performance of manufacturing processes. But a complete optimization of a manufacturing process requires a holistic approach, and adopting microgrid architectures can actually empower the optimization of a manufacturing process. In this paper, synergies about microgrids and manufacturing processes planning will be pointed out. Thus, a suitable approach to MG planning for manufacturing companies from a Knowledge Discovery in Databases (KDD) point of view is described.
\end{abstract}

\section{Keywords}

Industrial microgrid, planning, Knowledge discovery in databases, data mining

\section{Introduction}

Beyond the information age, it can be considered that we are nowadays involved in a new age that could be defined as the Internet age. This is a period in human history characterized by an economy based not only in the computerization of information, but also in knowledge linking and connection.

Internet of things (IoT) is nowadays one most important topics regarding computer-based science in business environments. This term arose about 1999, and many authors have approached it since then. For example F. Mattern and C. Floerkemeier asserts in [1] that IoT represents a vision in which the Internet extends into the real world embracing everyday objects. Physical items are no longer disconnected from the virtual world, but can be controlled remotely and can act as physical access points to Internet services.

As a result, it can be expected that the progressive connection of everyday objects to internet will be used to remotely determine their state so that information systems can collect up-to-date information on physical objects and processes [1]. Also devices are expected to communicate each other, and to develop a certain level of intelligence. Main objectives of the integration of this kind of devices are data gathering, measuring and communication. C. Perera et al. cite in [2] identify smart grid, smart homes and smart industries between main contributors to smart products sales market by 2016 .

The growing application of IoT-based strategies in manufacturing companies is a potential source of data for alternative energy supply systems planning, such as microgrids (MG). In addition, combined approaches to energy supply (using a MG architecture) and manufacturing process planning are expected to generate new optimization opportunities.

\section{About to KDD techniques}

Knowledge discovery in databases (KDD) can be described as the process of discovering useful knowledge from a collection of data. A. Berstein et al. also define $\mathrm{KDD}$ as the result of an exploratory process involving the application of various algorithmic procedures for manipulating data, building models from data, and manipulating the models. [3]

Since the grow of the amount of data in systems, no longer allows the manual search of underlying patterns, the main objective of KDD is to extract high-level knowledge from low-level information, or in other words, to automatically process large quantities of raw data, identify the most significant and meaningful patterns, and present these as knowledge appropriate for achieving the user's goals.[4].

Relationship between KDD, IoT and Data Mining (DM) is described in an accurate way by N. Ramakrishan in [5]:

- IoT collects data from different sources, which may contain data for the IoT itself.

- KDD, when applied to IoT, will convert the data collected by IoT into useful information that can then be converted into knowledge.

- DM is responsible for extracting patterns from the output of the data processing step and then feeding them into the decision making step, which takes care of transforming its input into useful knowledge. 
A growing body of emerging DM-based applications is changing the landscape of business decision support [6]: risk analysis, targeted marketing, customer retention[7], portfolio management and brand loyalty [8]. Traditional DM approaches are focused on modeling a variable of interest, so that this variable may be forecast in future scenarios, and effective decisions taken based on that forecast. These approaches are divided in [5] into four major categories based on how the information is extracted: predictive modeling (also called classification or supervised learning), clustering (also called segmentation or unsupervised learning), frequent pattern extraction and hybrid.

DM technologies are reviewed, described and by C. Tsai et al. [5]. They point out that most KDD systems available today and most traditional mining algorithms cannot be applied directly to process the large amount of data of IoT. In this line of work, they define three key considerations in choosing the applicable mining technologies for the problem to be solved by KDD techniques: objective, characteristics of data, and mining algorithm. In other words, whether or not to develop a new mining algorithm can be easily justified by using these factors.

Heuristic [9,10] [11] [12] and metaheuristic algorithms [13] are pointed out in [14] between trends in data mining algorithms applied to solve a wide variety of mining and optimization problems.

Perhaps one of the most important aspects in data mining problems is how to evaluate a candidate model, and, obviously, this question depends on the type of data mining task at hand. Thus, most of the data mining problems can be considered as optimization problems, where the aim is to evolve a candidate model that optimizes certain performance criteria. However, the majority of data mining problems have multiple criteria to be optimized. Hence, most of the data mining problems are multiobjective in nature. Multiobjective evolutionary algorithms for data mining are surveyed in [15] and [16] into different categories regarding the DM task they face.

Beyond specific trends in the field of data mining, KDD field is in continuous evolution towards the direction pointed out by K.Cios y L. Kurgan in [17]. The future of $\mathrm{KDD}$ and $\mathrm{DM}$ process models is in achieving overall integration of the entire process through the use of other popular industrial standards. Another currently very important issue is to provide interoperability and compatibility between different software systems and platforms, which also concerns KDD and DM models. Such systems would serve end-users in automating, or more realistically semi-automating, work with KDD systems.

\section{About to smart industries}

Factories of the future (FoF) is not only a motivational term related to the evolution of manufacturing process, but also a Public-Private Partnership under Horizon 2020, the EU Framework Programme for Research and Innovation.
This term concerns technological application towards manufacturing processes improvement basically in different research lines, addressing specific issues such as [18]:

- Modeling and simulation methods involving multiple phenomena such as physical, mechanical, energetic, chemical, energy, material characteristics, cost (...).

- Integrated knowledge-based systems covering the complete product life-cycle with advanced analytics, smart decision support systems and self-learning capabilities exploiting the availability of "big data" from smart sensors, historical process files, or humanauthored data; and addressing aspects (...).

- Integrated information management systems for product-process-production systems that are well embedded into their social, environmental and economic context.

- Advanced computer aided technologies, modeling, simulation and decision support toolboxes tailored for novel manufacturing processes.

Data acquisition, communication, and decision-making based on the acquired data are essential functions in modern manufacturing processes. That is the reason why $\mathrm{Z}$. Bi et al. affirms that the IoT is able to provide solutions to planning, scheduling, and controlling of manufacturing systems at all levels, due to the close relations of the components in a manufacturing enterprise and the architecture of IoT [19].

The growing use of condition-based monitoring is widely accepted (if not always implemented) by most manufacturers towards maintenance, production and energy management optimization.

Taking the idea a step further, manufacturers are looking forward to connect all these devices with higher decision-making levels[20]. This plant (or even multiple plant) connectivity from the device (or field) level to the enterprise (or decision-making) level connection involves connecting industrial devices to manufacturing execution systems (MESs), energy management systems (EMSs) or enterprise resource planning systems (ERPs).

Correspondingly the point of smart industries is not only to connect smart devices each other, but also to use gathered information in order to model and optimize a manufacturing process. This is the natural evolution of manufacturing management systems such as MES, EMS and ERPs.

In other words, KDD techniques are expected to take part of the future manufacturing management systems, assisting company managers along the decision-making processes. Data acquisition, communication and analysis are nowadays essential functions for the aforementioned MES, EMS and ERP systems.

Under this context, data use is expected to grow also in manufacturing processes. Several information from productive process variables remains in data bases, which at the end, store huge amounts of historical data. As a consequence, KDD techniques have many potential applications in the field of manufacturing processes optimization and decision making.

Hence, quality improvement (QI) programs including 
Six Sigma, and Kaizen require collection and analysis of data to solve quality problems. Due to advances in data collection systems and analysis tools, DM has widely been applied for QI in manufacturing[21]. The structure of a specific data mining model for synthetic ammonia decarbonization process optimization and the application of fuzzy rules to automatic prediction of several qualityrelated attributes of steel coils manufacturing process are described in [22] and [23].

Not only quality but also energy requirements for manufacturing processes have been addressed with data mining techniques. Energy supply has risen in importance last decade due to the raise of energy costs for industrial companies. EMSs are one of the most trending IoT applications to manufacturing processes nowadays. EMSs are based on ICTs and software tools for tracking energy use and identifying savings opportunities. Since they are based in energy consumption information, gathered by energy measurement equipment such as power analyzers or flow meters at the flied level, this systems are expected to evolve quickly to forecasting, modeling and optimizing applications.

Not only general but also specific DM applications have been developed with energy saving proposes. The research on the process control and the energy saving of the aluminum electrolysis industry have inspired new specific data mining algorithms [24]. These algorithms provide intelligence to a novel enhanced-control model which consists of the following four main modules: the aluminum electrolysis structure data mining module, the time series data mining module, the risk forecast module and the online mining module.

A. Kusiak and Z. Shong describe the existence of three different approaches to optimization of combustion efficiency: analytical models based on thermodynamics and chemistry, soft computing and hybrid systems [25]. Optimization data-mining based models for improvement of a boiler-turbine system performance are formulated in [26] and [27]. These papers are focused on optimizing performance indexes (such as the unit heat rate and the total fuel consumption) and discussing optimal selection of process variables to optimize combustion efficiency.

A framework for the analysis of process mining algorithms is described in [28]. A process discovery algorithm is compared in this paper with a learning machine, whose task is to model the control flow of a business process, using traces of the execution of the process, recorded in a workflow $\log$ which is a multiset over traces.

Traditional DM and machine learning (ML) algorithms make predictions on the future data using statistical models that are trained on previously collected labeled or unlabeled training data. But since traditional ML techniques try to learn each task from scratch, other new approaches like transfer learning techniques try to transfer the knowledge from some previous tasks to a target task when the latter has fewer high-quality training data [29]. Based on the definition of transfer learning, S.Pan and Q.Yang summarize the relationship between traditional ML and various transfer learning settings and categorize transfer learning under three subsettings, inductive transfer learning, transductive transfer learning, and unsupervised transfer learning.

There is no doubt that new techniques and systems enable new KDD problems approaches. Many researchers all over the world are doing great efforts for optimizing or even revolutionizing the existing techniques, tools and systems. In the prospect of the further requirements, some new requirements together with problems will emerge with the further increasing amount and widespread application of data for manufacturing companies. Demand for real-time processing, on-demand processing, as well as the intransit processing of standard remote sensing data products are some of the development opportunities [30] in this field.

\section{A KDD approach to microgrid planning}

Large (and growing) data volumes are being continuously created by energy-related industrial (or even domestic) equipment. This data can be used with different purposes from improving energy efficiencies to developing new services for energy consumers.

Following this guidelines, KDD approaches to $\mathrm{MG}$ planning could be developed. This concept can be applied not only to improve electrical networks performance but also to intelligently manage the actions of all the systems connected to them (those who generate electricity, who use electricity and who take both actions). Therefore, KDD, DM and IoT techniques are expected to drive the evolution of power systems into smart systems. Moreover, the visions of smart grids and IoT have recently been combined into the Internet of Energy (IoE)[31].

Furthermore, data analysis from a manufacturing process towards different objectives such as process control, quality assurance and environmental impact evaluation is a powerful source of knowledge towards industrial processes planning.

As a consequence, during a downstream planning stage, production and energy supply could be studied in and aggregated way with saving purposes for a company.

The availability of a big amount of data from every stage of the systems can reveal several opportunities to improve manufacturing process efficiencies at every level: energy generation, energy distributions, energy consumption, manufacturing global process efficiency, manufacturing equipment efficiency and occupation. This is, without any doubt, a suitable framework for industrial MG planning.

Recent approaches to MG planning for industrial facilities have been published in technical literature. B Leif et al. in [32] propose a technique for sizing and scheduling electricity supply at industrial sites with combined heat and power and wind generation. An industrial size MG is scheduled in [33] and evaluated regarding different performance indicators such as technical, economic and environmental. The techno- 
economic potential for a predominantly renewable electricity-based MG serving an industrial-sized drink water plant in the Netherlands is studied in [34] by $M$ Soshinskaya et al.

But among all the articles reviewed in order to write this paper, only M. Overturf et al. in [35] combine MG (energy supply) an manufacturing process (production) planning for industrial companies. They developed a Kaizen-based approach (which they call Sufficiency Kaizen) and conclude that taking control over the allocation and production of energy, corporations can obviate energy cost impact and risk, with relief immediate, and absolute impact over time.

In conclusion, synergies of an aggregated planning approach to manufacturing processes and MG-based energy supply have not been deeply analyzed. This new approach to MG planning is expected to reveal both energy and manufacturing cost saving opportunities, increasing economic feasibility and reliability for both systems.

\section{Industrial MG planning future trends}

The full potential of energy saving for a manufacturing process can only be defined through a holistic and integrated analysis of the complete value chain of the plant. In this approach, a $\mathrm{MG}$ can be considered as a suitable energy supply system, and data analytics can provide information about how systems work in a day-today (even hour-by hour) basis.

MG planning have been traditionally approached from investment minimization [36] and economic load dispatch problems. But beyond economic goals, other different goals can be considered along the planning process, such as total environmental impact, power quality and reliability [37]. Regarding technical literature there exist some defined problems in every MG planning process, even though each microgrid planning process has its own constraints and specific goals. Different power supply optimization opportunities appear at different MG planning levels, such as

- Power generation mix selection and sizing: Microgrid design engineers are responsible of choosing the best available power system to satisfy demand requirements. Power sources selection requires a deep site analysis of suitable electric power supplies. Power [38] and energy storage equipment [39] must be sized according peak load demand and cost effectiveness criteria. Types of fuels suitable for the power plant must also be selected, which is a critical issue regarding cost efficiency and reliability of the system.

- Siting problem covers power sources allocation and power lines layout in order to keep quality constraints [38].

- Scheduling problem is aimed at minimizing operational costs, environmental impact and quality keeping while demand is covered.

KDD techniques application to industrial MGs involves a different approach to these problems, since a large amount of raw data about the manufacturing process is available. This new focus results in new cost-saving opportunities. Some of them are described below

\section{A. New sizing approach}

Main goals of sizing stage in a MG planning process are high cost-effectiveness, low power losses impact and high reliability. According to these guidelines, power and energy storage equipment must be sized in order to cover peak load demand and to achieve cost effectiveness. Some KDD-based opportunities approaches to these problems are described below:

- New bottom up-approach. since traditional MG problems are focused on defining and covering peak load (simply adding total power capacity an estimating a simultaneity factor), KDD approach is able to make a real bottom-up approach to peak demand due to the existing knowledge in manufacturing energy consumption data. The analysis of these data makes possible to define different power demand steps regarding different usage scenarios for manufacturing equipment. Some different coverage strategies can be developed defining different sizes and regulation capacities for power supply equipment. Optimization models can be validated with real data from the manufacturing process.

- Redesigning energy systems: the availability of raw data about the manufacturing process is a clear advantage in order to redesign the power supply system with new objectives such as renewable power sources penetration, ancillary services supply, net metering capacities and combined heat and power integration. This revision should be focused in economic feasibility of the MG but also other goals such as environmental impact or quality keeping can be considered.

- Capacity charge savings: a MG can considerably reduce capacity charge costs from utilities. In order to achieve these savings, an optimal mix of power technologies and power capacities must be defined both for MG. These power capacity reduction savings are based again into power consumption data analysis (from the manufacturing process). A detailed costbenefit analysis on power capacity must be done in order to achieve a long term economic viability for the MG.

\section{B. New siting approach}

Quality keeping is the main goal of siting problems in MG planning. The previous existence, not only of data, but also of the manufacturing facilities process is a clear advantage. Quality problems will be for sure well-defined since they cannot be only modeled but also measured. In other words, the capacity and allocation requirements both for storage and power generation systems with quality keeping purposes can be validated using on site field measurements. Hence, siting problem will consider real conditions and for sure the solution will be more accurate.

\section{New scheduling approach}

Perhaps the most powerful source of cost savings in $\mathrm{KDD}$ approach to $\mathrm{MG}$ planning is on resources 
scheduling. The aggregated scheduling of manufacturing and energy supply resources can reduce global manufacturing process costs. Some guidelines to be followed towards this goal are described below:

- Integration of energy generation variables into industrial management systems: as it has been mentioned before the future of systems such as ERPs and EMSs is in integration and analytics. A combined analysis tool both for energy generation, energy consumption and manufacturing conditions will allow the company to identify new scheduling-based performance improvement scenarios. But not only analytics and management tool can reveal saving opportunities.

- Forecasting and simulation: real data from a manufacturing process is the basis of a robust manufacturing process model development. Once this model is developed, forecasting capabilities have proven to be very useful for scheduling tasks and savings achievement. Simulation is also an important tool in order to find optimal work conditions for power supply and manufacturing equipment. Both tools are needed in order to define the most efficient working parameter for the whole system.

These techniques will allow innovative specific approaches such as:

- Energy supply and manufacturing combined scheduling: a combined planning for manufacturing process and energy resources is expected to achieve cost saving. This savings will mainly be based on waste energy minimization, following this guidelines:

$\checkmark$ Coordinated starts and stops not only for the manufacturing process but also for the power equipment.

$\checkmark$ Power generation regulation according to manufacturing process requirements.

- Advanced energy efficiency strategies: some advanced strategies such as net metering and demand response must be studied in order to generate profits to the company. Some of this opportunities will derive on automatic control strategies, price signals conditioned energy purchase or sell of energy from other power grids, cost managing. All of this energy-related strategies must be verified regarding production costs in order to assure economic revenues for the whole process.

\section{Conclusions}

MG systems are suitable to provide energy to manufacturing processes, even though it is an energy intensive consumption field. $\mathrm{MG}$ and manufacturing processes have many goals in common regarding quality, environmental impact, cost reduction and energy efficiency. Companies quantify these goals using MES, EMS and ERPs, which are based on specific indicators also called Key Performance Indicators (KPIs).

Nowadays, MES, EMS and ERPs are a powerful source of data about manufacturing processes. The application of KDD techniques to the industrial field allows the merge of production and energy management.
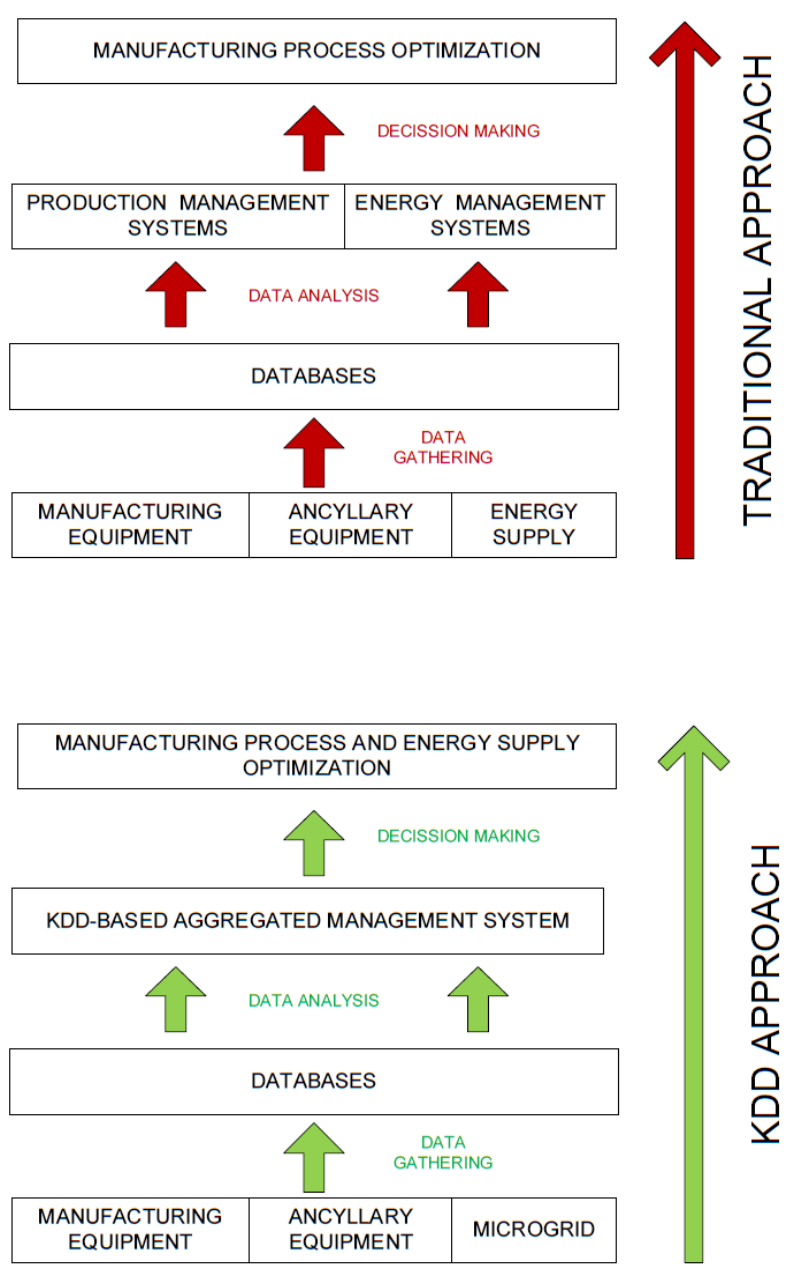

Fig. 1. Differences between traditional and KDD planning approaches for manufacturing processes.

Thus, energy experts using data analytics techniques are able to discover and exploit hidden saving opportunities, thereby contributing effectively in making faster, better and quality business decisions. The full potential of energy saving for a manufacturing process can only be defined through a holistic and integrated analysis of the complete value chain of the plant. Following this guideline, an innovative KDD-based approach to industrial MG planning has been introduced in this paper.

\section{References}

[1] Mattern F, Floerkemeier C. From the internet of computers to the internet of things. Lect. Notes Comput. Sci. (including Subser. Lect. Notes Artif. Intell. Lect. Notes Bioinformatics), vol. 6462 LNCS, 2010, p. 242-59. doi:10.1007/978-3-642-17226-7 15.

[2] Perera C, Liu CH, Jayawardena S, Chen M. A Survey on Internet of Things From Industrial Market Perspective. IEEE Access 2014;2:1660-79. doi:10.1109/ACCESS.2015.2389854.

[3] Bernstein A, Provost F, Hill S. Toward intelligent assistance for a data mining process: An ontology-based approach for cost-sensitive classification. IEEE Trans Knowl Data Eng 2005;17:503-18. doi:10.1109/TKDE.2005.67.

[4] Matheus CJ, Chan PK, Piatetsky-Shapiro G. Systems for knowledge discovery in databases. IEEE Trans Knowl Data 
Eng 1993;5:903-13. doi:10.1109/69.250073.

[5] Tsai CW, Lai CF, Chiang MC, Yang LT. Data mining for internet of things: A survey. IEEE Commun Surv Tutorials 2014;16:77-97. doi:10.1109/SURV.2013.103013.00206.

[6] Gandomi A, Haider M. Beyond the hype: Big data concepts, methods, and analytics. Int $\mathrm{J}$ Inf Manage 2015;35:137-44. doi:10.1016/j.ijinfomgt.2014.10.007.

[7] Xiang Z, Schwartz Z, Gerdes JH, Uysal M. What can big data and text analytics tell us about hotel guest experience and satisfaction? Int J Hosp Manag 2015;44:120-30. doi:10.1016/j.ijhm.2014.10.013.

[8] Apte C. Data mining: an industrial research perspective. IEEE Comput Sci Eng 1997;4:6-9. doi:10.1109/99.609825.

[9] Li J. On optimal rule discovery. IEEE Trans Knowl Data Eng 2006;18:460-71. doi:10.1109/TKDE.2006.1599385.

[10] Espejo PG, Ventura S, Herrera F. A survey on the application of genetic programming to classification. IEEE Trans Syst Man Cybern Part C Appl Rev 2010;40:121-44. doi:10.1109/TSMCC.2009.2033566.

[11] Díaz Arevalo JL, Pérez García R. Estado del arte en la utilización de tecnicas avanzadas para la busqueda de información no trivial a partir de datos en los sistemas de abastecimiento de agua potable n.d.

[12] Shin CK, Yun UT, Kim HK, Park SC. A hybrid approach of neural network and memory-based learning to data mining. IEEE Trans Neural Netw 2000;11:637-46. doi:10.1109/CDC.2014.7039892.

[13] De La Iglesia B, Reynolds A. The use of metaheuristic algorithms for data mining. Proc 1st Int Conf Inf Commun Technol ICICT 2005 2005;2005:34-44. doi:10.1109/ICICT.2005.1598541.

[14] Kulkarni R V, Member S, Förster A, Venayagamoorthy GK. Computational Intelligence in Wireless Sensor Networks: A Survey. Commun Surv Tutorials, IEEE 2011;13:68-96. doi:10.1109/SURV.2011.040310.00002.

[15] Mukhopadhyay A, Maulik U, Bandyopadhyay S, Coello CAC. A survey of multiobjective evolutionary algorithms for data mining: Part i. IEEE Trans Evol Comput 2014;18:4-19. doi:10.1109/TEVC.2013.2290086.

[16] Mukhopadhyay A, Maulik U, Bandyopadhyay S, Coello C a C. Survey of multiobjective evolutionary algorithms for data mining: Part II. IEEE Trans Evol Comput 2014;18:2535. doi:10.1109/TEVC.2013.2290082.

[17] Cios KJ, Kurgan LA. Advanced Techniques in Knowledge Discovery and Data Mining. 2005. doi:10.1007/184628-183-0.

[18] European Commision. Call FoF February 2015. Web Page 2015.

http://ec.europa.eu/research/participants/portal/desktop/en/opport unities/h2020/topics/2187-fof-08-2015.html (accessed February 20, 2015)

[19] $\mathrm{Bi} \mathrm{Z}, \mathrm{Xu} \mathrm{L} \mathrm{Da}$, Wang C. Internet of things for enterprise systems of modern manufacturing. IEEE Trans Ind Informatics 2014;10:1537-46. doi:10.1109/TII.2014.2300338.

[20] Dutta D, Bose I. Managing a big data project: The case of Ramco Cements limited. Intern J Prod Econ 2014. doi:10.1016/j.ijpe.2014.12.032.

[21] Köksal G, Batmaz I, Testik MC. A review of data mining applications for quality improvement in manufacturing industry. Expert Syst Appl 2011;38:13448-67. doi:10.1016/j.eswa.2011.04.063.

[22] Gao L-HGL-H, Luo S-XLS-X, Qiu J-QQJ-Q, Li FCLF-C, Ran H-CRH-C. Data mining on a kind of complex industrial process. Proc 2003 Int Conf Mach Learn Cybern (IEEE Cat No03EX693) 2003;1:105-7. doi:10.1109/ICMLC.2003.1264451.

[23] Krömer P, Platos J, Snášel V. Mining multi-class industrial data with evolutionary fuzzy rules. 2013 IEEE Int Conf Cybern CYBCONF 2013:191-6.
doi:10.1109/CYBConf.2013.6617453.

[24] Zhuo CZC, Bingtu YBY, Yinglong WYW, Zefeng SZS. A new model for the industrial process control based on Data Mining. 2008 Chinese Control Decis Conf 2008:1368-70. doi:10.1109/CCDC.2008.4597541.

[25] Kusiak A, Song Z. Combustion efficiency optimization and virtual testing: A data-mining approach. IEEE Trans Ind Informatics 2006;2:176-84. doi:10.1109/TII.2006.873598.

[26] Song Z, Kusiak A. Constraint-based control of boiler efficiency: A data-mining approach. IEEE Trans Ind Informatics 2007;3:73-83. doi:10.1109/TII.2006.890530.

[27] Kusiak a., Zhe Song. Clustering-Based Performance Optimization of the Boiler\&\#x2013;Turbine System. IEEE Trans Energy Convers 2008;23:651-8. doi:10.1109/TEC.2007.914183.

[28] Weber P, Bordbar B, Tiño P. A Framework for the Analysis of Process Mining Algorithms.pdf. IEEE Trans Syst Man Cybern Syst 2013;43:303-17. doi:10.1109/TSMCA.2012.2195169.

[29] Pan SJ, Yang Q. A survey on transfer learning. IEEE Trans Knowl Data Eng 2010;22:1345-59. doi:10.1109/TKDE.2009.191.

[30] Ma Y, Wu H, Wang L, Huang B, Ranjan R, Zomaya A. Remote sensing big data computing: Challenges and opportunities. Futur Gener Comput Syst 2014. doi:10.1016/j.future.2014.10.029.

[31] Bui N, Castellani AP, Casari P, Zorzi M. The internet of energy: A web-enabled smart grid system. IEEE Netw 2012;26:39-45. doi:10.1109/MNET.2012.6246751.

[32] Leif Hanrahan B, Lightbody G, Staudt L, Leahy PG. A powerful visualization technique for electricity supply and demand at industrial sites with combined heat and power and wind generation. Renew Sustain Energy Rev 2014;31:860-9. doi:10.1016/j.rser.2013.12.016.

[33] Bracco S, Delfino F, Pampararo F, Robba M, Rossi M. A mathematical model for the optimal operation of the University of Genoa Smart Polygeneration Microgrid: Evaluation of technical, economic and environmental performance indicators. Energy 2014;64:912-22. doi:10.1016/j.energy.2013.10.039.

[34] Soshinskaya M, Crijns-graus WHJ, Meer J Van Der, Guerrero JM. Application of a microgrid with renewables for a water treatment plant. Appl Energy 2014;134:20-34. doi:10.1016/j.apenergy.2014.07.097.

[35] Overturf MC, Chrosny WM, Stine R. Energy Sufficiency Kaizen Achieving Zero Energy Cost and Variance with Sufficiency-inclusive Private Energy Portfolios. Distrib Gener Altern Energy J 2011;26:36-56. doi: $10.1080 / 21563306.2011 .10462203$.

[36] Asano H, Bando S. Economic evaluation of microgrids. IEEE Power Energy Soc 2008 Gen Meet Convers Deliv Electr Energy 21st Century, PES 2008:1-6. doi:10.1109/PES.2008.4596603.

[37] Morris GY, Abbey C, Wong S, Joos G. Evaluation of the costs and benefits of Microgrids with consideration of services beyond energy supply. IEEE Power Energy Soc Gen Meet 2012:1-9. doi:10.1109/PESGM.2012.6345380.

[38] Vallem MR, Mitra J. Siting and sizing of distributed generation for optimal microgrid architecture. Proc. 37th Annu. North Am. Power Symp. 2005, vol. 2005, 2005, p. 611-6. doi:10.1109/NAPS.2005.1560597.

[39] Sun C. Sizing of hybrid energy storage system in independent microgrid based on BP neural network. 2nd IET Renew Power Gener Conf (RPG 2013) 2013:3.45-3.45. doi:10.1049/cp.2013.1851. 